Recibido: marzo de 2018

Aprobado: mayo de 2018

DOI: https://doi.org//0.15332/rev.m.v15i0.2177

Meccanismo di collasso per scivolamento nelle piattabande. Basílica del Socorro

Fonte: Michele Paradiso, 2017.

Reporte de caso. Conservazione de patrimonio storico costruito latinoamericano. Dipartimento di Architettura, DiDA - Università degli Studi di Firenze (Italia). [Conservación del patrimonio histórico construido en Latinoamérica]

* Profesor asociado de Rehabilitación Estructural de Patrimonio Histórico Construido, Departamento de Arquitectura DiDA - Universidad de los Estudio de Florencia, Italia; miembro de: Icomos-Cuba; IcoFort/lcomos; Iscarsah/lcomos. Lleva 40 años investigando sobre el comportamiento estructural de arcos y bóvedas de mampostería, y además en técnicas sustentables de consolidación de monumentos históricos. Correo electrónico: michele.paradiso@unifi.it

** Arquitecto de la Universidad Santo Tomás Bucaramanga (Colombia); especialista en Planificación del Territorio para el Desarrollo. Politécnico di Milano (Italia) - Université de Paris I, Pantheon-Sorbonne, París (Francia); MSc en Relaciones Internacionales y Estudios Estratégicos con énfasis en Políticas de Desarrollo. Lancaster Uinversity (Inglaterra), y PhD en Planificación Urbana, Territorial y Ambiental - Politécnico di Milano (Italia). Docente investigador, líder de la línea de investigación en Planificación y Gestión del Territorio adscrita al grupo de Investigaciones de la Facultad de Arquitectura de la Universidad Santo Tomás Bucaramanga (Colombia). Correo electrónico: carlos. gomez@ustabuca.edu.co

\section{LA BASILICA MINORE DI NUESTRA SEÑORA DEL SOCORRO E IL SUO GRADO DI SICUREZZA STRUTTURALE*}

\author{
Michele Paradiso** - Università degli Studi di Firenze, Italia \\ Carlos Humberto Gómez Arciniegas*** - Universidad Santo Tomás, Colombia
}
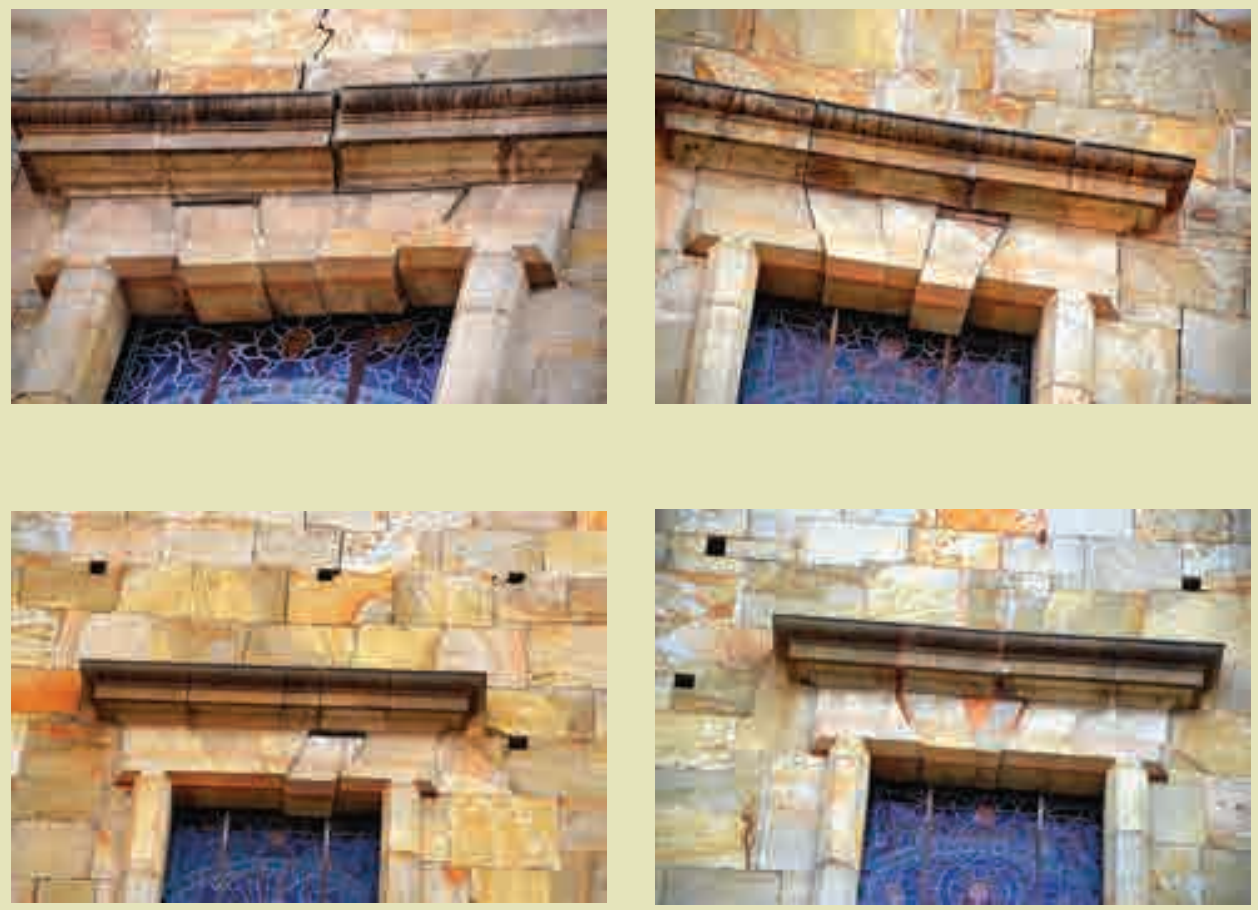

\section{RIASSUNTO}

L'articolo si riferisce allo stato di degrado strutturale della Basilica Minore della città di Socorro nella Regione di Santander (Colombia). La Concattedrale di Socorro, recentemente elevata al rango di Basilica Minore, ha meno di un secolo di vita e presenta un quadro fessurativo critico a tal punto da sollecitare la massima urgena per gli interventi di salvaguardia. Dopo un breve escursus storico e la descrizione dell'impianto architettonico, si descrive nel dettagli il quadro fessurativo, alla data del marzo 2017. La Concattedrale presenta un tipico meccanismo di collasso che consiste nello scivolamento verso il basso dei conci dei grandi archi e delle piattabande delle grandi aperture sui fronti estreni. Tale tipo di meccanismo é ascrivibile ad azioni di tipo cinematico, riconducibili a cedimenti fondali. Sopratutto si raccontano le vicende che hanno accompagnato, dal novembre 2016 al maggio 20I7, un tentativo, poi fallito, di intervento tecnico da parte delle istituzioni preposte alla salvaguardia del bene, dichiarato peraltro monumento nazionale, fallimento dovuto, in base alle conclusioni della ricerca effettuata, alla mancanza di un piano strategico di salvaguardia del patrimonio storico del Socorro.

\section{PAROLE CHIAVE}

Conservazione, restauro, Socorro, meccanismo di collasso. 


\section{BASÍLICA MENOR DE NUESTRA SEÑORA DEL SOCORRO Y SU GRADO DE SEGURIDAD ESTRUCTURAL}
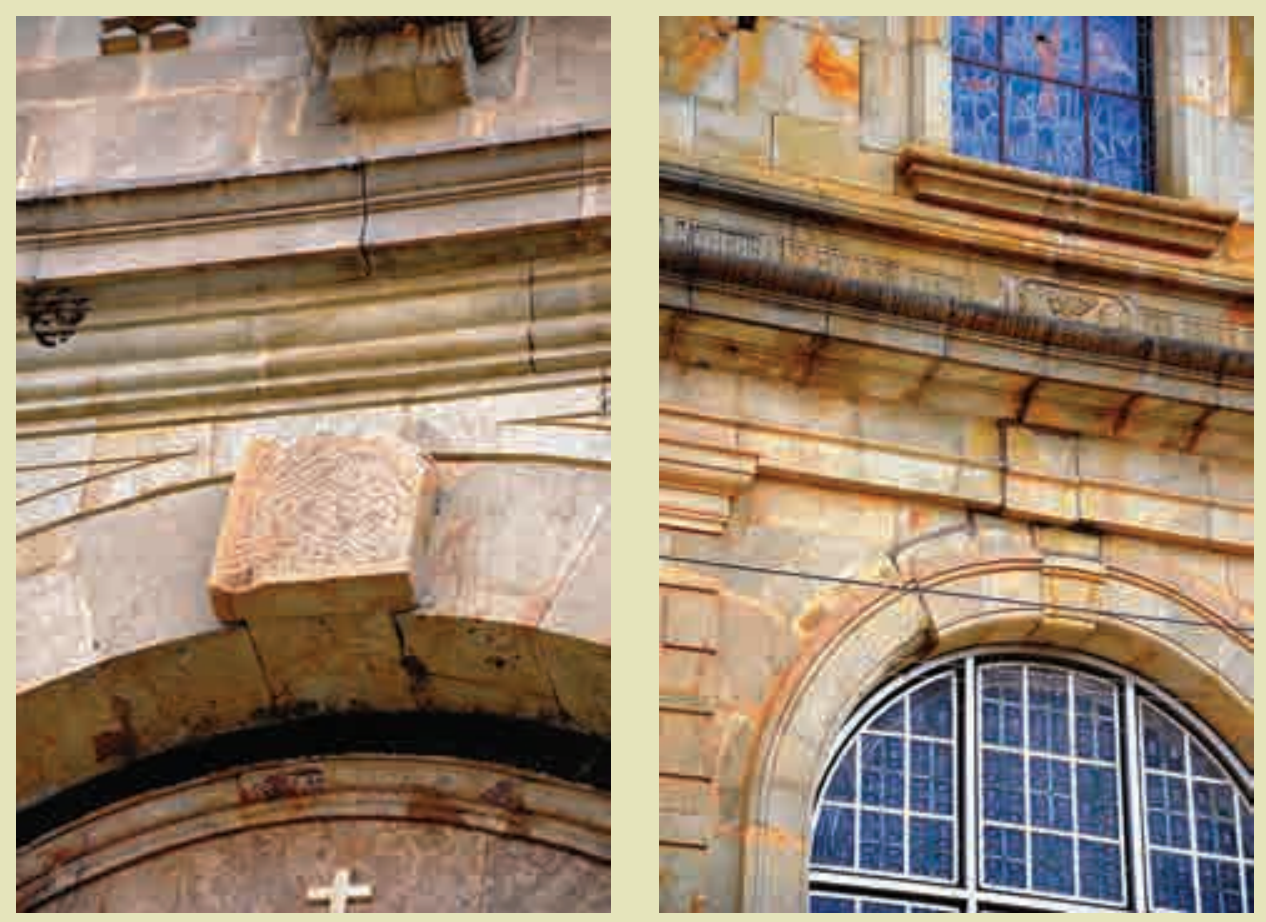

Mecanismo de colapso por desplazamientos de las dovelas de los arcos

Fonte: Michele Paradiso, 2017.

\section{RESUMEN}

El artículo se refiere al actual estado de deterioro estructural de la Basílica Menor de la ciudad del Socorro en el departamento de Santander (Colombia). La Concatedral de Socorro, recientemente elevada al rango de Basílica Menor, tiene menos de un siglo de antigüedad y presenta un patrón crítico de grietas que hacen que se requieran con carácter de urgencia intervenciones de salvaguarda. Después de un breve recorrido histórico y la descripción del objeto arquitectónico, se describe en detalle la situación de las grietas hasta marzo de 2017. La Concatedral presenta un mecanismo de colapso típico que consiste en el deslizamiento hacia debajo de las dovelas que componen los grandes arcos así como de las platabandas sobre las fachadas. Este tipo de mecanismo es atribuible a acciones de tipo cinemático, probablemente relacionadas a hundimientos del suelo. Así bien, se hace énfasis en las diferentes situaciones que han acompañado, desde noviembre de 2016 a mayo de 2017, los tentativos de intervención técnica por parte de las instituciones responsables de salvaguardar el inmueble, declarado monumento nacional, catalogado como fallidos con base en las conclusiones de la investigación realizada, a causa de la falta de un plan estratégico para salvaguardar el patrimonio histórico del Socorro.

\section{PALABRAS CLAVE}

Conservación, restauración, Socorro, mecanismo de colapso. 


\section{CENNI STORICI SULLA CITTÀ DI SOCORRO}

Socorro, come è noto, venne fondata 16 giugno 1683 . Si distingue per la sua architettura coloniale e per essere stata nella storia l'epicentro della rivoluzione dei Comuneros nel I 78I. E' il capoluogo della provincia comunera e una delle prime città fondate nel Nuovo Regno di Granada, dove è iniziato il percorso che ha portato all'indipendenza della Colombia. Il centro della città, il suo cuore storico, è testimonianza del passato che si può ancora riconoscere grazie alle sue piccole chiese coloniali, all'imponente Basilica, alle moltissime emergenze di carattere storico e a un assetto urbano che rispetta ancora i canoni urbanistici delle antiche Leggi delle Indie. Nell'anno I78I, la Villa de el Socorro apparteneva alla Provincia di Tunja con le città di Tunja, Muzo, Velez, Girón, Pamplona, Salazar de las Palmas e Villa de Leiva. Occupò il quarto posto per popolazione dopo Santa Fe, Cartagena e Popayán. Epicentro commerciale della regione, le sue industrie e i suoi manufatti furono i più grandi nel Nuovo Regno di Granada.

La fondazione di Socorro non è avvenuta secondo la prassi dei conquistadores, che vedevano nel nuovo mondo la possibilità di fondare la ciudad ideal, sia dal punto di vista urbanistico che architettonico. L'origine della piccola città fu molto più prosaica e semplice. A grandi linee, si può dire che si è formata lungo la strada che portava da Vélez a Girón, nel luogo esatto in cui si trova oggi. Don José de Archila e Don José Díaz Sarmiento, ricchi proprietari terrieri della valle del Chanchón, donarono parte dei loro possedimenti alla Vergine di Nostra Signora di Socorro, allo scopo di fondare la nuova città. Decine di anni dopo, Socorro ricevette l'importante riconoscimento di Villa muy noble y mu leal, riconoscimento con il quale il re Carlo III di Spagna (17|6- |788), cercava di migliorare il sentimento spagnolo nelle colonie. Era l'anno I77I. Più tardi, il 25 aprile I773, fu concesso lo stemma, obbedendo all'esaltazione mariana. Nel I 795 il re Carlo IV (I748 - I8I9), creò la provincia di Socorro con la capitale dallo stesso nome, con un territorio superiore alla metà dell'attuale Dipartimento di Santander, provincia che rimarrà in vigore fino al 1857 , quando il Congresso della Repubblica fondò lo Stato di Santander che in seguito fu sovrano. I primi decenni del diciannovesimo secolo videro emergere i movimenti indipendentisti nelle colonie spagnole dell'attuale Colombia. Secondo Arciniegas (1992), “...nella Nuova Granada la guerra di indipendenza, ha cominciato a germogliare nel Socorro...”, a causa delle eccessive tasse imposte dalla corona spagnola. Noto come il Comunero, nacque un periodo di rivoluzione contro le autorità coloniali, praticamente l'unica insurrezione sociale documentata negli annali patriottici. Nel villaggio di Socorro molte delle sue strade, edifici e piazze sono ancora testimoni della Rivoluzione Comunera con tanti eventi storici che hanno dato a questa popolazione un posto di rilievo nella storia colombiana. Guadagnata la libertà dalla Corona Spagnola, la città di Socorro nel I86I divenne la capitale del Dipartimento di Santanderi. Venticinque anni più tardi perse questo privilegio a favore di Bucaramanga.

\section{IL CENTRO STORICO DI SOCORRO A LA SUA BASILICA}

II centro storico di Socorro risente negativamente dell'intenso traffico automobilistico, di un commercio disordinato, di una speculazione edilizia che preferisce evitare di conservare il proprio patrimonio storico sostituendolo, isolato per isolato, con edilizia moderna di valore estetico assolutamente discutibile, mal interpretando il significato e le scelte conseguenti del dilemma tipico dell' America Latina sullo sviluppo sostenibile dei centri storici: renovar o conservar.

II centro fu dichiarato patrimonio di interesse culturale nazionale, mediante Decreto 264 del 1963 (Fig. I). 


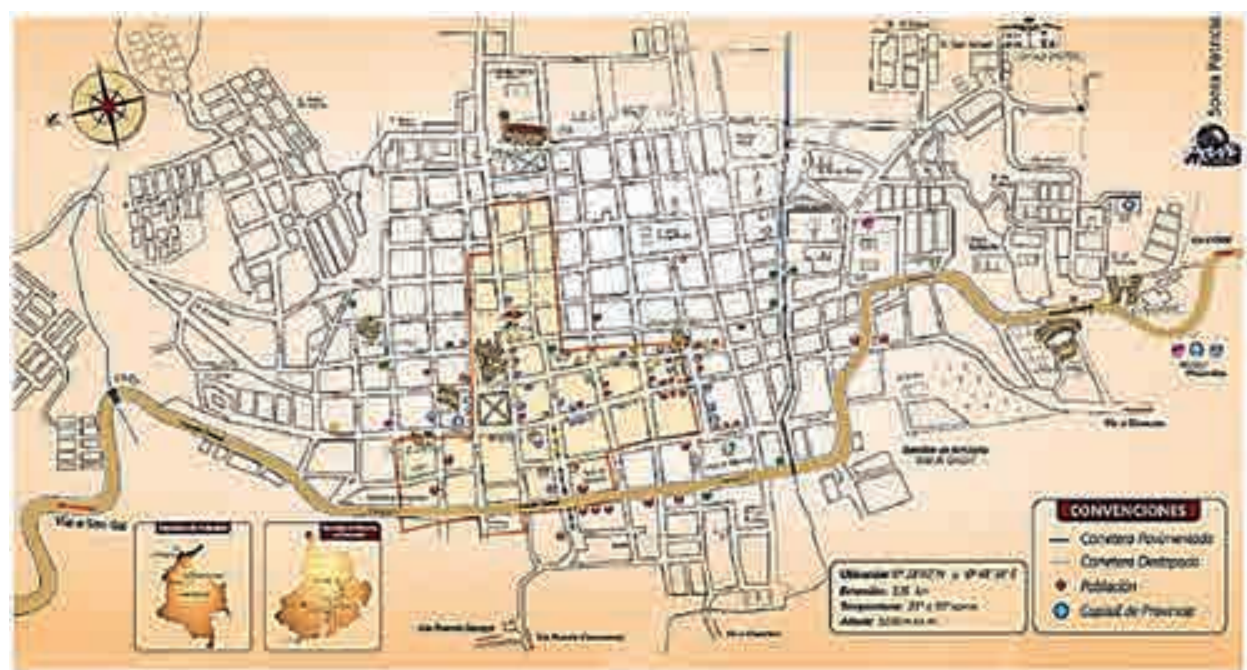

Inoltre, nell'ambito del Piano Nazionale per il Recupero dei Centri Storici (PNRCH), nel 2005, il Ministero della Cultura ha stanziato le risorse per l'appalto della prima fase del Piano di Protezione Speciale (PEP) di Socorro, attualmente gestito dal Dipartimento di Santander, il cui obiettivo é quello di consolidare il centro urbano con proposte volte a recuperare ed esaltare il suo enorme valore patrimoniale.

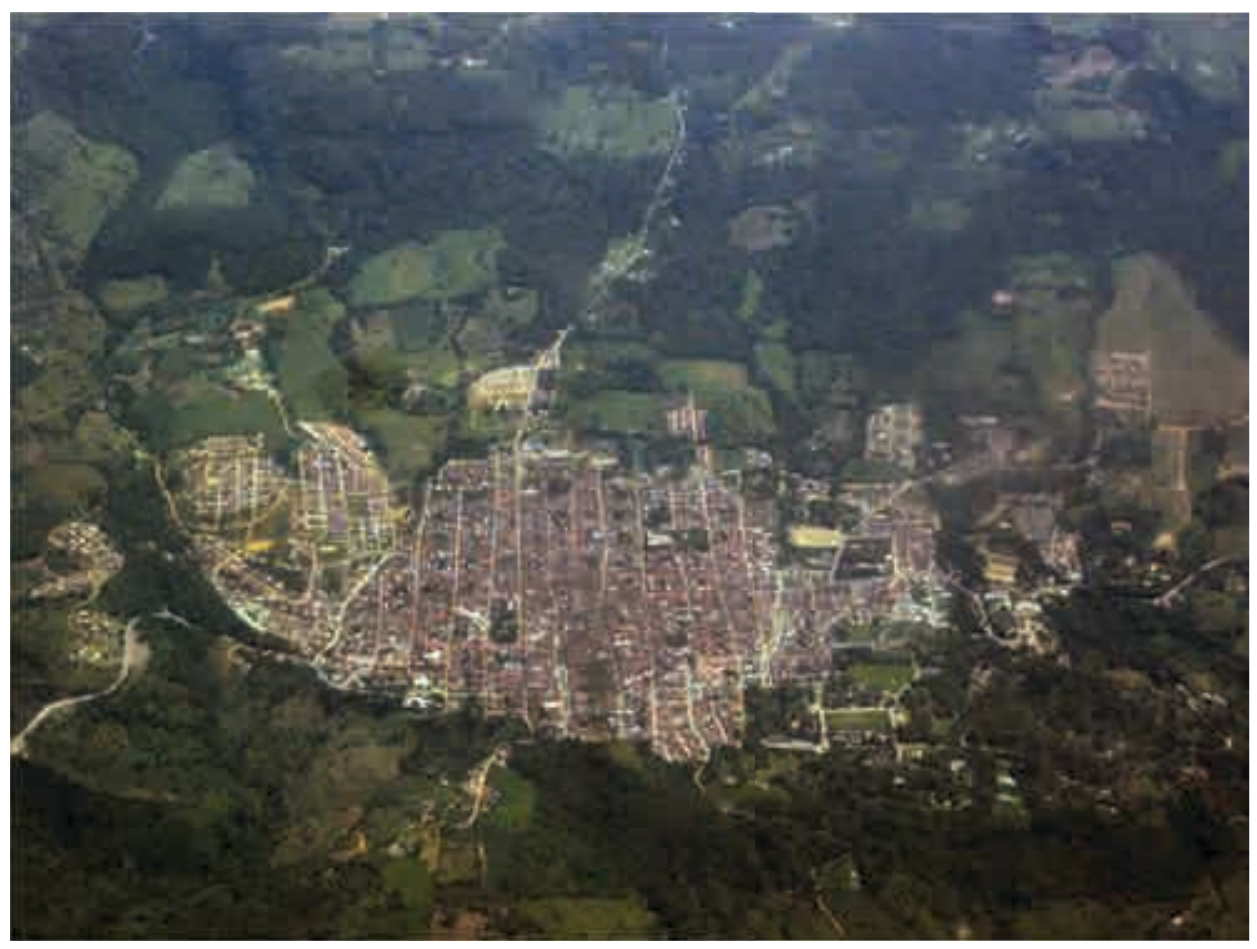

Nel nucleo centrale del paese, prospiciente alla parco principale della città (Parque de la Independencia, dove fu uccisa l'eroina della Indipendenza Antonia Santos), sorge un monumento di grande importanza: la Basilica Minore di Nostra Signora del Socorro. La sua costruzione ebbe inizio nel 1873 sotto la direzione di Bonifacio Vargas e lgnacio Martínez. Si tratta di una cattedrale monumentale posizionata sui due assi principali del tessuto urbano del comune. La Basilica si trova su un lato della piazza principale della città di Socorro, esattamente su uno degli angoli (Fig. 3).
Figure I. Mappa del Centro Storico di Socorro. Si evidenzia l'area del centro storico. dichiarata patrimonio di interesse culturale. Fonte: http://elsocorro-santander.blogspot. com.co/p/datos-generales.html

Figure 2. Veduta aerea di Socorro. E evidente la caratteristica reticola urbana degli insediamenti spagnoli della Colonia. Fonte: www.mapio.net. 
Figure 3. Ubicazione della Basilica Minore di Nostra Signora di Socorro nel nucleo del centro storico del paese La mappa consente di stabilire la corretta posizione Nord-Sud della Chiesa

Fonte: Adattazione degli autori in base a Google Earth.
Figure 4. Facciata della Basilica Minore di Nostra Signora di Socorro Fonte: Carlos Gómez, 2017.

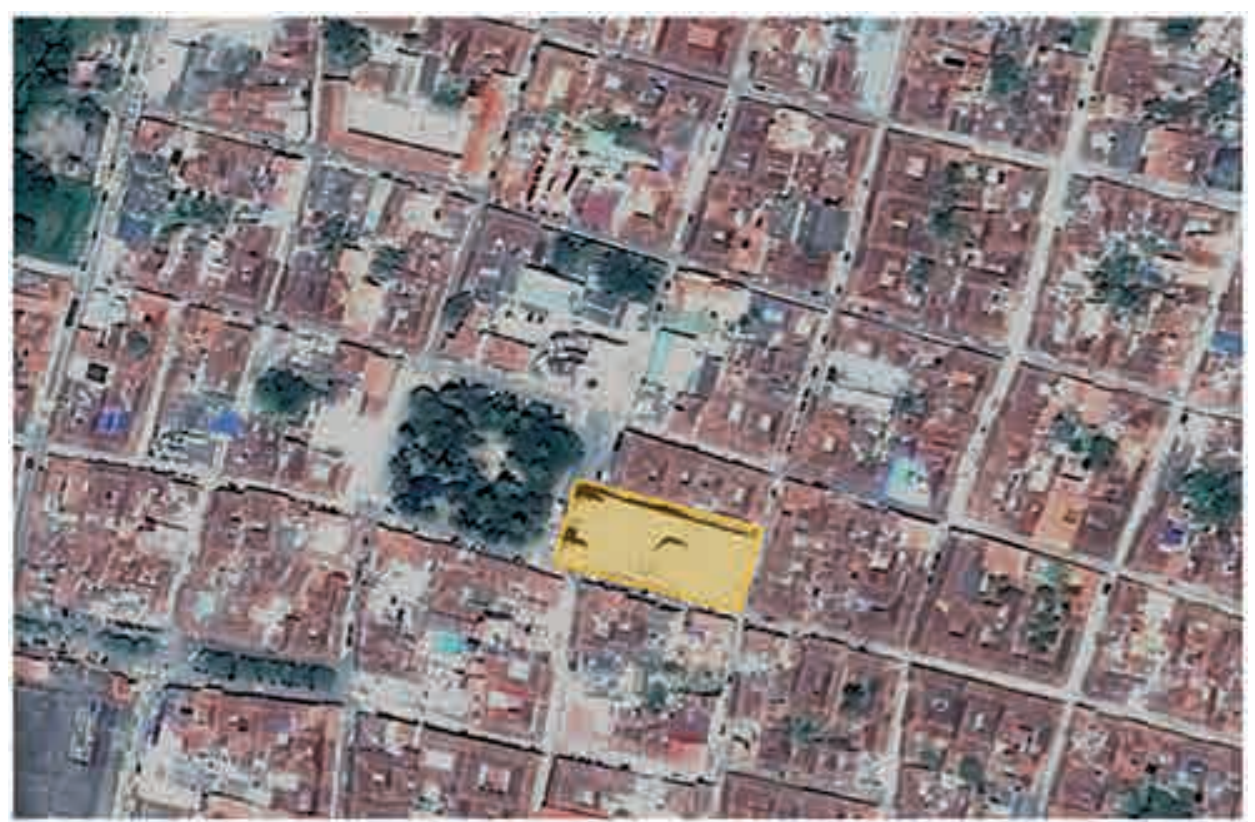

Mediante la legge 267 del 20I I, il Senato della Repubblica ha dichiarato la Basilica di Nuestra Señora del Socorro (di Santander) bene di interesse culturale della Nazione: con l'occasione furono pubblicate disposizioni per la conservazione del bene.

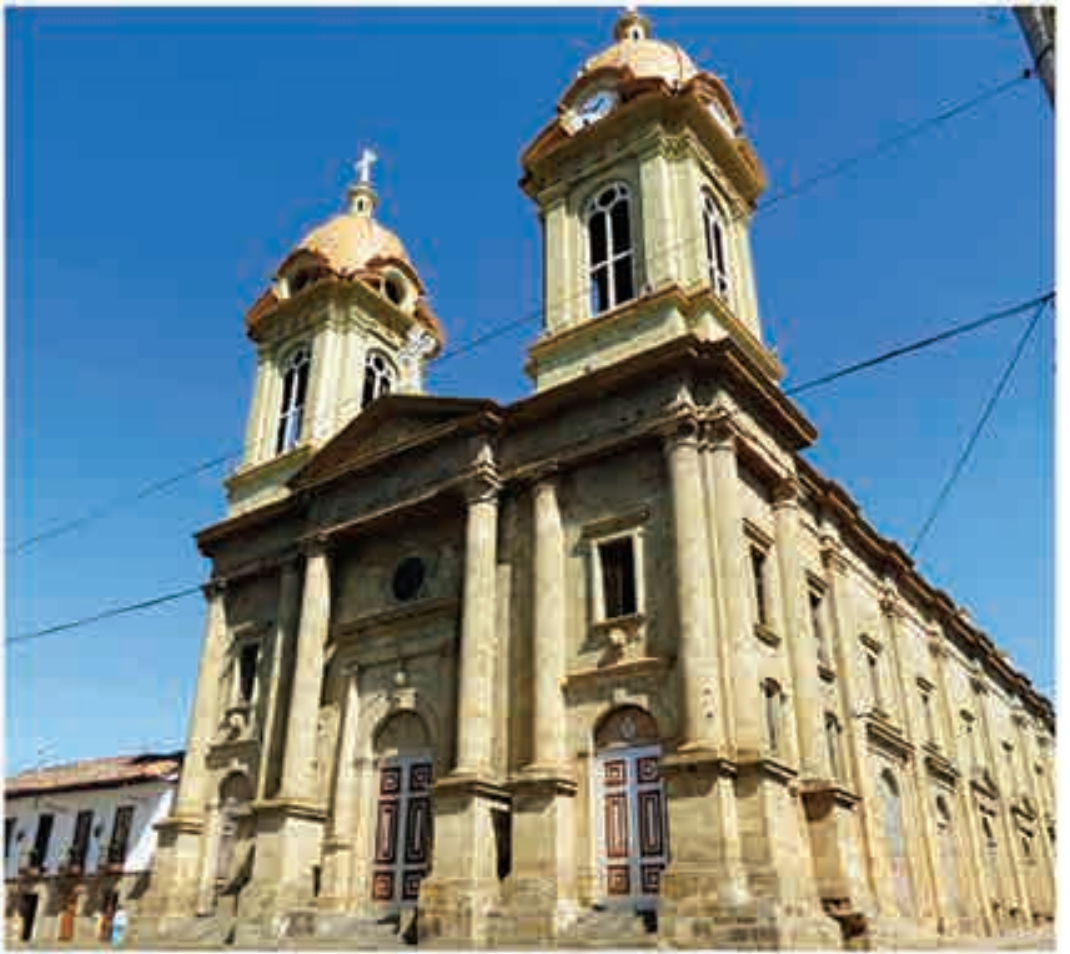

Come già detto, la costruzione della cattedrale ebbe inizio il I 4 dicembre I 873 a carico di Bonifacio Vargas, un modesto artigiano (oggi si direbbe un maestro pedreiro) e da Ignacio Martínez. Fu terminata ufficialmente l'I I giugno 1943, grazie alla partecipazione diretta di benefattori locali, Ad ogni modo i lavori di abbellimento proseguirono fino al dicembre 1954. I progettisti del tempo inserirono nella chesa molti richiami sia al Rinascimento Italiano sia alla monumentalità del neoclassicismo europeo (Fig. 5). Tuttavia la chiesa è definita 
essere in stile repubblicano, in omaggio ai canoni della storia dell'architettura colombiana e latinoamericana. Un fatto importante della storia è proprio la dedizione all'impresa di Bonifacio Vargas che, nonostante il suo figliuolo e discepolo avesse perduto la vita proprio nel cantiere per un incidente sul lavoro, guidò la costruzione fino al suo termine, destreggiandosi egregiamente nella costruzione sia delle robuste mura come delle esili colonne e degli gli archi carpanel (arco policentrico ribassato), Racconta la leggenda come fosse una opera ammirata dalla ingegneria civile di allora e come un gruppo di studenti della Scuola di ingegneria di Bogotà, sotto la guida del noto Architetto José Ramón Peña e dell'ingegnere Carlos Camargo, avessero successivamente provveduto al rilievo planimetrico del monumento, per lasciare testimonianza storica della sua eccellenza. Probabilmente per mancanza di finanziamenti, nei primi anni ' 30 il cantiere subì una battuta d'arresto: il caso della Chiesa incompiuta divenne una caso nazionale dibattuto socialmente fino a che non intervenne direttamente il Governo assegnando per il suo completamento un finanziamento speciale (legge 53 del 1927).

\section{IL DEGRADO STRUTTURALE DELLA BASILICA}

La Basilica Minore di Nuestra Señora del Socorro, già Cattedrale (per decreto di Papa Leone XII, il 20 marzo 1895), poi Concattedrale, è catalogata come la più grande costruzione in pietra scolpita della Colombia, con una mescolanza di pietra di Barichara (Colombia) e marmo di Carrara (Italia). II titolo di Basilica Minore venne concesso dalla Santa Sede a partire dal 16 giugno del 2015 (Decreto 409-14 expedido por la Congregación para el Culto Divino y la Disciplina de los Sacramentos), rispondendo positivamente alla richiesta inviata in Vaticano l'anno precedente, dal Vescovo di Socorro e San Gil, competente per giurisdizione.

Da un artcolo di Vanguardia.com del 16 aprile 2015:

... El obispo Carlos Germán Mesa Ruiz dio a conocer apartes del documento con fecha del 16 de marzo de 2015, del decreto protocolo 409-14 expedido por la Congregación para el Culto Divino y la Disciplina de los Sacramentos, entidad encargada de responder la 'solicitud enviada hace un año en el mes de junio, para acceder al título de Basílica Menor a la Concatedral Nuestra Señora del Socorro'.

Según el Obispo por cumplir con todos los requisitos exigidos, el señor Cardenal Prefecto, Roberto Sarah, y el arzobispo secretario Arturos Rosch, reconocen este título 'con todos los derechos y ritos católicos que se podrán ejercer en este hermosísimo templo'. Mesa Ruiz dijo que la Basílica desde hace varios años viene siendo muy bien cuidada, "he visto la dedicación que los sacerdotes y vicarios han tenido con la Basílica Menor, hace un tiempo se dio el cambio de párroco, fue nombrado el padre Juvenal Landínez, 'él siguió con la tarea de embellecimiento, de cuidado del templo, ustedes han sido testigos de que cada vez está mejor presentada, y pensando en que esta solicitud sería aprobada, entonces nos adelantamos a los acontecimientos de embellecer nuestra Concatedral, y responder ahora con mayor razón al título que se hará oficial el día de Nuestra Señora del Socorro que se celebra el 16 de junio, para esta fecha se llevará a cabo una ceremonia especial de reconocimiento'. El Obispo indicó que en los próximos días se dará inicio a una serie de intervenciones en la parte física del templo para cumplir con los requisitos exigidos por el Vaticano, al ser designada como Basílica Menor esta reliquia arquitectónica. El alcalde de Socorro, Humberto Corzo Galvis, dijo que "es una excelente noticia para los socorranos, ya que esta exaltación va a tener una connotación importante a nivel nacional, máxime cuando hacemos parte de la red de pueblos patrimonio de Colombia.

M 33 REVISTA M VOL. 15 ENERO - DICIEMBRE 2018 - FACULTAD DE ARQUITECTURA • UNIVERSIDAD SANTO TOMÁS COLOMBIA 
La autorización de Roma de elevar la Concatedral a Basílica Menor se oficializará mediante un acto solemne el próximo 16 de junio, pues en esa fecha se conmemora un año más de la vida institucional del Socorro.

En la parte turística cada vez somos más importantes y se espera que cada día lleguen más turistas a la capital comunera, dijo el Alcalde...

Abbiamo voluto riportare tesualmente le parole dell'Obispo e dell'Alcalde per sottolineare come le due istituzioni preposte alla conservazione del bene, dal punto di vista religioso e laico, nelle loro dichiarazioni lasciano intendere come abbiano ben presente significato e importanza del concetto di conservazione del bene e giustamente lo leghino allo sviluppo economico locale, attraverso l'attrazione di sempre più visitatori e turisti nazionali e internazionali. L'Obispo in particolare sostiene di avere sembre posto la massima attenzione alla cura e all'imbellimento del monumento, e annuncia l'inizio di una serie di interventi tecnici fortemente suggeriti addirittura dal Vaticano per dare concretezza alla cura che si deve a un monumento dichiarato Basilica Menor.

Purtroppo la realtà risulta abbastanza diversa e la situazione dell'edificio, dal punto di vista strutturale, si porta dietro problemi che, a parere degli autori, se non risolti con la massima rapidità, potranno mettere a serio rischio la sicurezza del Monumento. La Basilica Menor de Socorro, infatti, già da troppi anni presenta un complesso e notevole quadro fessurativo: le lesioni tagliano il corpo dell'edificio secondo tutti i piani paralleli in direzione trasversale al corpo di fabbrica e in corrispondenza delle campate delle tre navi interne. Partcolarmente si nota la tendenza al distacco dei volumi della parte anteriore, che comprende le due torri campanarie, dalla parte restrostante. Le lesioni piu' prossime alla facciata risultano piu' gravi, diminuendo in ampiezza mano a mano che si proceda verso la parte tergale. Per spiegare meglio il quadro fessurativo analizziamo dapprima la configurazone geometrica della Basilica, facendo riferimento alle tavole di rilievo sotto riportate, per gentile concessione della Secretería de Cultura del Departamento Santander. L'impianto chiesistico é a tre navate, coperte con volte a crociera, prevalentemente ribassate e quindi fortemente spingenti. Allo scopo di descrivere poi il quadro fessurativo, chiameremo le tre navate: centrale, laterale sinistra, laterale destra, immaginando di porci di fronte alla facciata della Chiesa; In senso longitudinale chiameremo invece le otto ripartizioni, campate, numerandole da sinistra a destra, da I a 8, immaginando di porci questa volta a guardare la facciata laterale in Calle 14. Sulla prima campata insistono le due massiccie torri campanarie. La luce di questa prima campata risulta minore delle altre per evidenti ragioni di sicurezza strutturale che i costruttori avevano ben presente, data la forte sismicità della zona: la campata più stretta garantiva una spinta minore, a fronte del forte peso delle torri, sugli archi di scarico delle corrispondenti crociere sottostanti. L'impianto architettonico, secondo le indicazioni della Chiesa Cattolica del tempo, non doveva prevedere accanto al monumento null'altro sen non la Casa Cural: per questo motivo la Chiesa si affaccia lateralmente su una strada, Calle 14, mentre dall'altro lato guarda principalemente al chiostro della Casa Cural. Inoltre la stessa Calle I 4 presenta una accentuata pendenza verso la Piazza antistante la Chiesa. Fatto comune in molti altri casi, uno tra tutti la Chiesa di Barichara ma anche la stessa Chiesa di Santa Lucia in Guane-Barichara. II materiale utilizzato per le murature portanti é la pietra locale, caliza, con peso specifico intorno ai 2000 $\mathrm{kg} / \mathrm{mc}$, con media ma significativa presenza di porosità. Tutto questo considerato, con solo considerazioni sull'impianto architettonico, e tenendo conto della forte sismicità della zona, oltreché della forte pressione sul tereno trasmessa dalle torri campanarie rispetto alle restanti murature di elevazione, la zono più intrinsecamente debole appare essere quella prospiciente all'angolata tra Carrera 14 e Calle I4.

Si riportano di seguito gli sviluppi dei quadri fessurativi più gravi facendo riferimento allo schema sottostante di numerazione delle campate. 


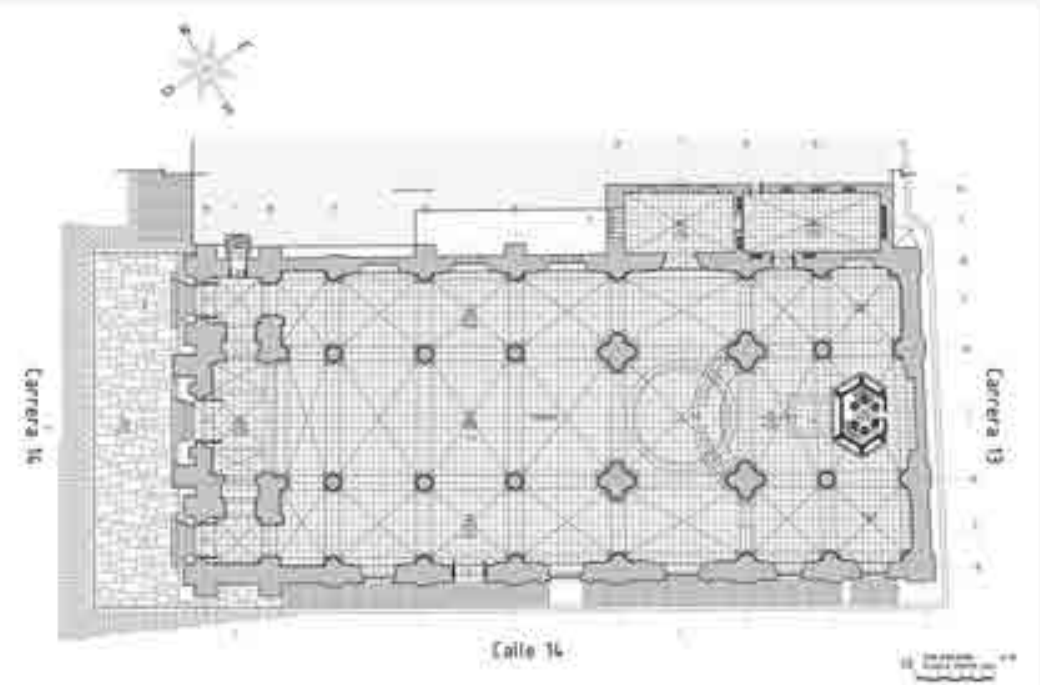

Figure 5. Pianta della Basilica Fonte: Ministerio de Cultura.
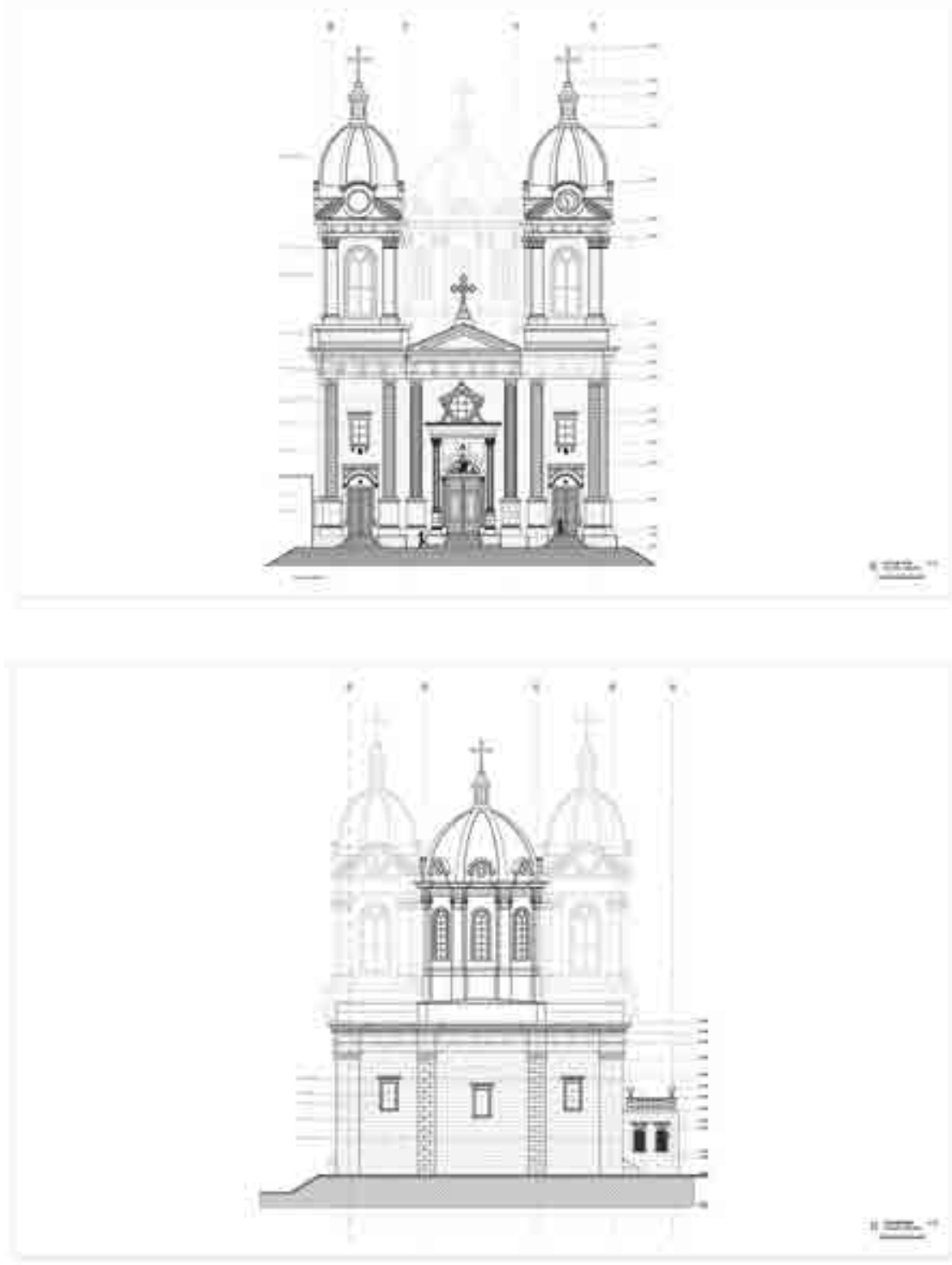
Figure 8. Prospetto laterale su Calle I4 Fonte: Ministerio de Culturo.

Figure 9. Prospetto-sezione lato Casa Cural Fonte: Ministerio de Cultura.

Figure 10. Sezione longitidinale B-B Fonte: Ministerio de Cultura.
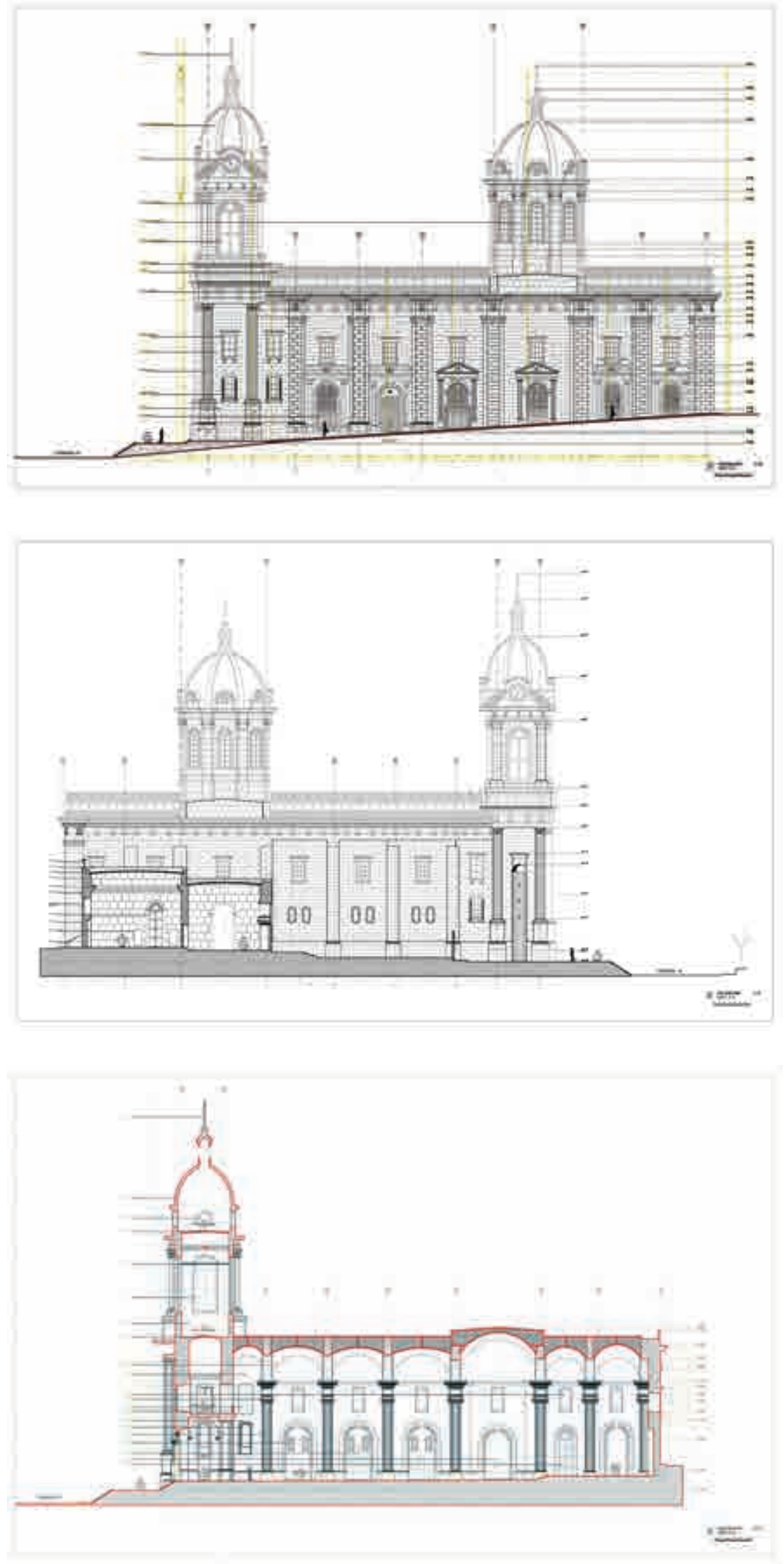

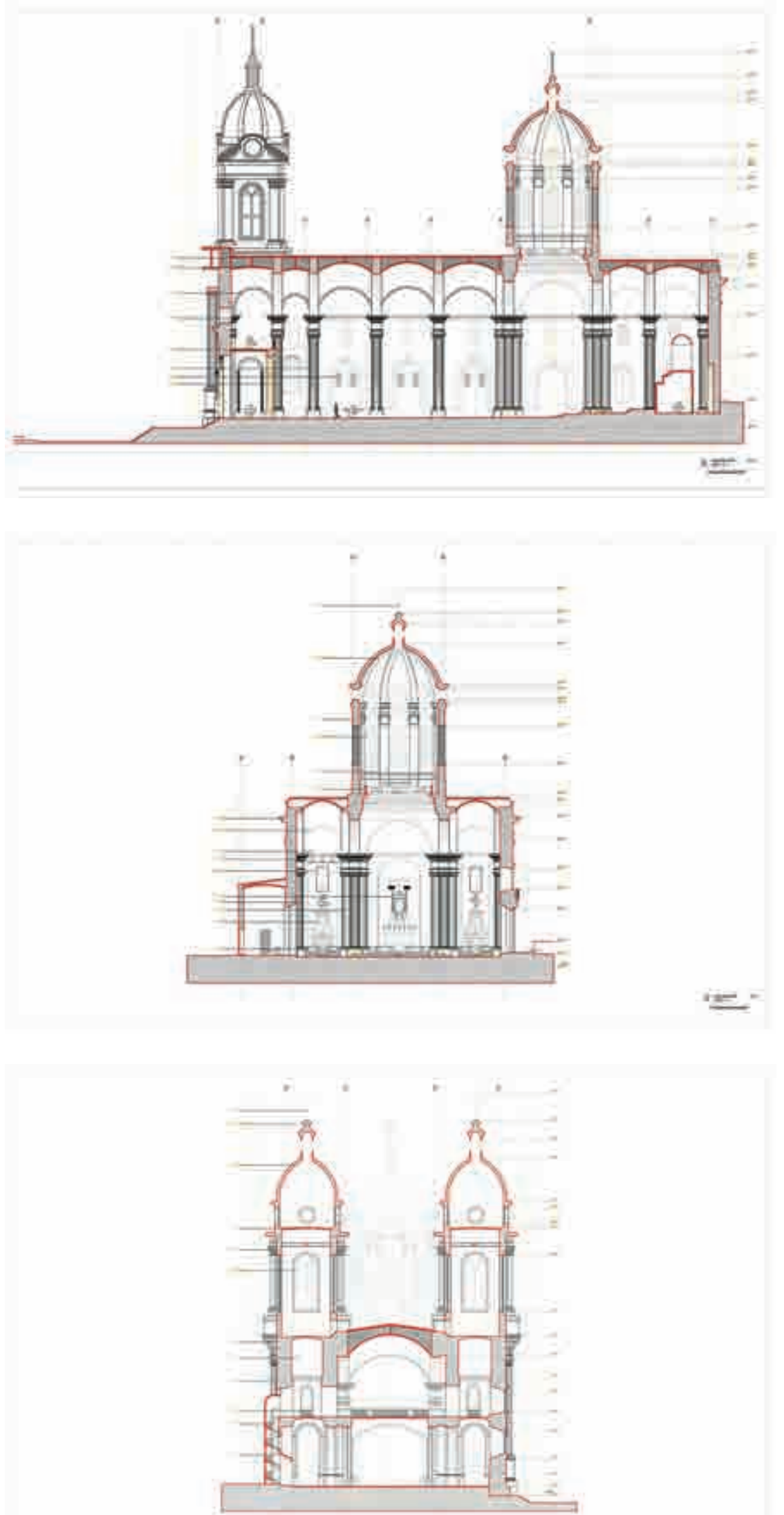

Figure II. Sezone longitudinale C-C Fonte: Ministerio de Culturo.

Figure 12. Sezione trasversale I-I Fonte: Ministerio de Cultura. 
Figure 14. Numerazione delle campate Fonte: Ministerio de Cultura, elaborazioni grafiche per Michele Paradiso.

Figure 15. Campata I, esterno, lato Calle 14 Fonte: Elaborazione grafica a cura di Olimpia Lotti e Antonio Farigu.

Figure 16. Campata I, esterno, lato Casa Cural Fonte: Elaborazione grafica a cura di Olimpia Lotti e Antonio Farigu.
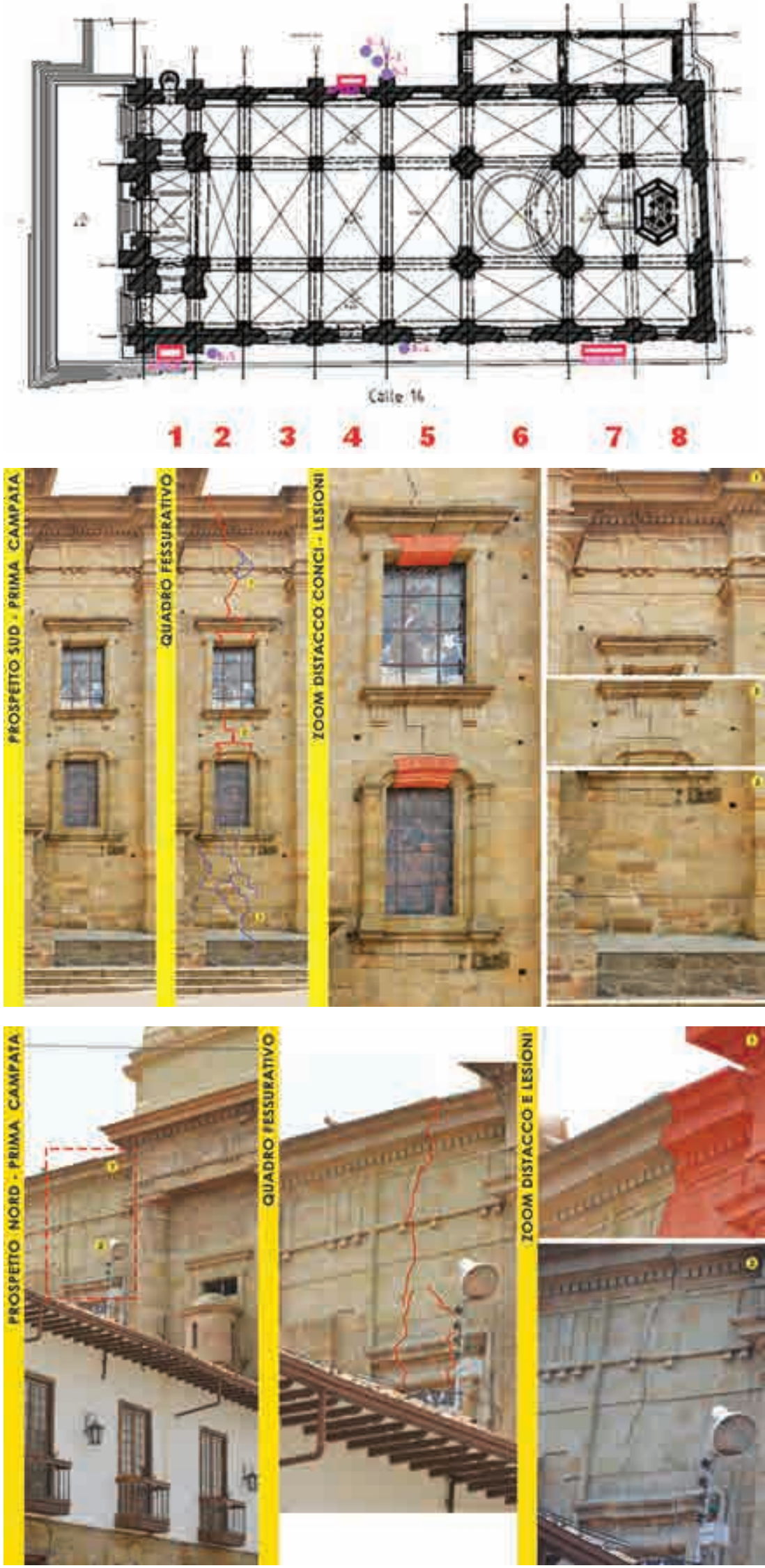

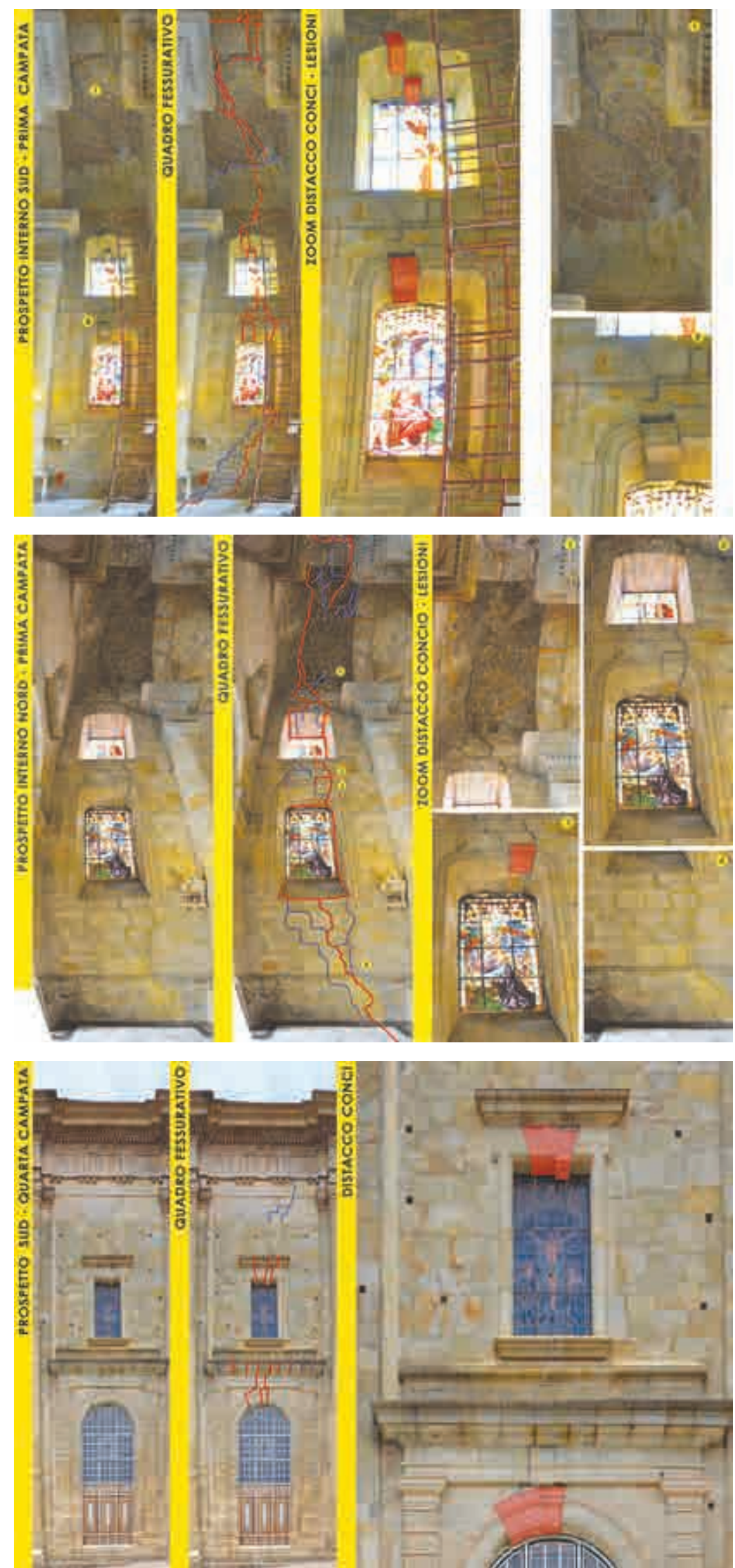

Figure 17. Campata I, interno, lato Calle I4 Fonte: Elaborazione grafica a cura di Olimpia Lotti e Antonio Farigu.
Figure 18. Campata I, interno, lato Casa Cural

Fonte: Elaborazione grafica a cura di Olimpia Lotti e Antonio Farigu.
Figure 19. Campata 3, esterno, lato Calle 14 Fonte: Elaborazione grafica a cura di Olimpia Lotti e Antonio Farigu. 
Figure 20. Campata 5, esterno, lato Calle 14 Fonte: Elaborazione grafica a cura di Olimpia Lotti e Antonio Farigu.

Figure 21. Panoramica interna del quadro fessurativo della campata I

Fonte: Elaborazione grafica a cura di Olimpia Lotti e Antonio Farigu.
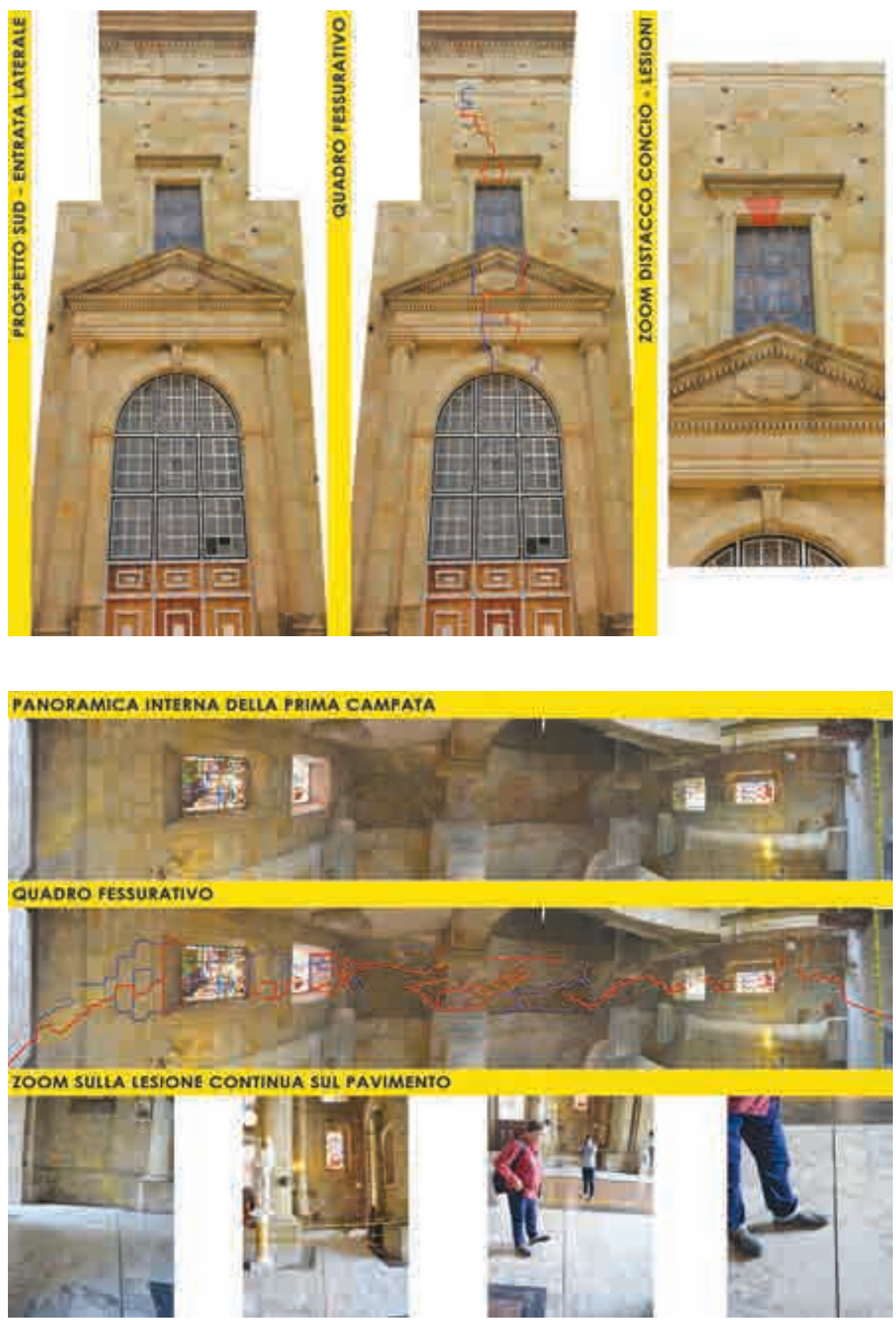

II rilevo del quadro fessurativo evidenzia quanto già accennato in precedenza: le lesioni sulle facciate laterali interessano l'intera altezza delle pareti, tagliano trasversalmente le volte a crociera e interessano anche la pavimentazione: di fatto la facciata della Chiesa è interessata da un grave cinematismo che tende a separarla dal corpo di fabbrica retrostante. I quadri fessurativi delle campate retrostanti ribadiscono questo fenomeno anche se la gravità tende a scemare verso la campata 8 , ribadendo il caratteristico fenomeno "a denti di pettine". L'andamento delle lesioni sulle pareti di elevazione è prevalentemente verticale con accennate presenze di tratti curvilinei a curvatura inversa (andamento a gola rovescia). Per interpretare questo quadro fessurativo, che di fatto parla da sé nella sua gravità, si può far riferimento in letteratura tecnica agli studi dell'Ingegnere italiano. Sisto Mastrodicasa (I887-1983), autore del volume "Dissesti statici delle strutture edilizie", nel quale Mastrodicasa fornisce ai tecnici un metodo che porti facilmente alla individuazione 
della cause dei dissesti, particolarmente per le strutture storiche in muratura, attraverso lo studio geometrico dell'andamento delle lesioni e un percorso analitico a ritroso della classica sequenza azioniesterne-sollecitazioniinterne-statotensionale-statodeformativo-dissesti (Mastrodicasa, 20I2). D'altra parte è ben noto ed è di usuale pratica, tenere presente a questo scopo che:

- In riferimento al materiale, il quadro fessurativo:

I. Risente delle caratteristiche meccaniche del materiale;

2. Nasce in corrispondenza di instabilità locali del tessuto materico:

- In riferimento alla struttura muraria, il quadro fessurativo:

3. Cerca i punti di debolezza interna della struttura, cioè i domini pluriconnessi, quali aperture di qualsiasi tipo;

4. Risente della natura del terreno, della consistenza del sistema fondale, della umidità di risalita per capillarità, delle forti e frequenti piogge, della azione sismica.

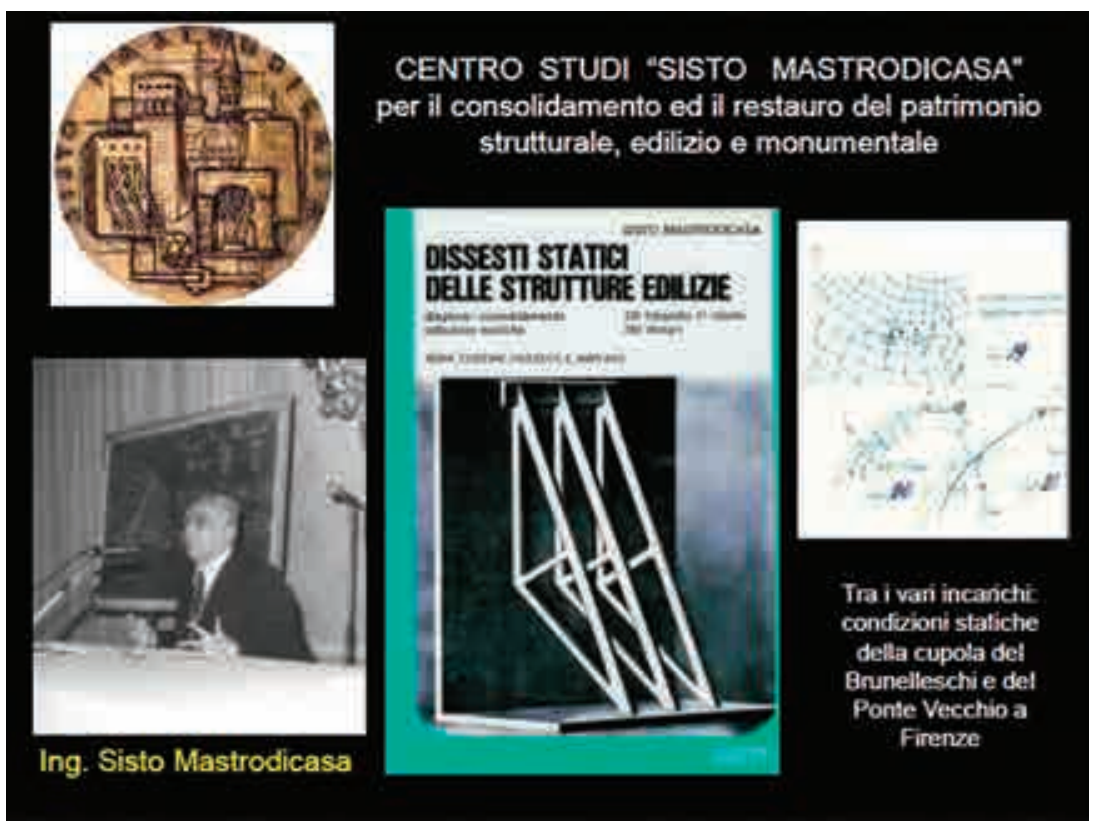

Figure 22. La Fondazione Sisto Mastrodicasa, Perugia-Italia

Fonte: Materiale didattico Michele Paradiso.

Non c'é alcun dubbio quindi che il complesso quadro fessurativo fin qui mostrato porti alla iniziale conclusione che non sono certamente azioni statiche a causarne il degrado, ma azioni cinematiche prima che azioni dinamiche di tipo sismico.

Infine, in questo articolato quadro, va posta particolare attenzione ai quadri fessurativi delle grandi architravi e degli archi delle grandi finestrature esterne. SI faccia riferimento alla Figure 23. Sia nel caso dell'arco di sinistra (facciata della Basilica) sia in quello di destra, si nota un pericoloso scivolamento dei conci della zona di chiave, con fenomeno di rototraslazione in avanti. 
Figure 23. Meccanismo di collasso per "cuneo" degli archi della Basilica

Fonte: Michele Paradiso, 2017.

Figure 24. Meccanismo di collasso "per cuneo" delle arco-travi (piattabande) Fonte: Michele Paradiso, 2017.
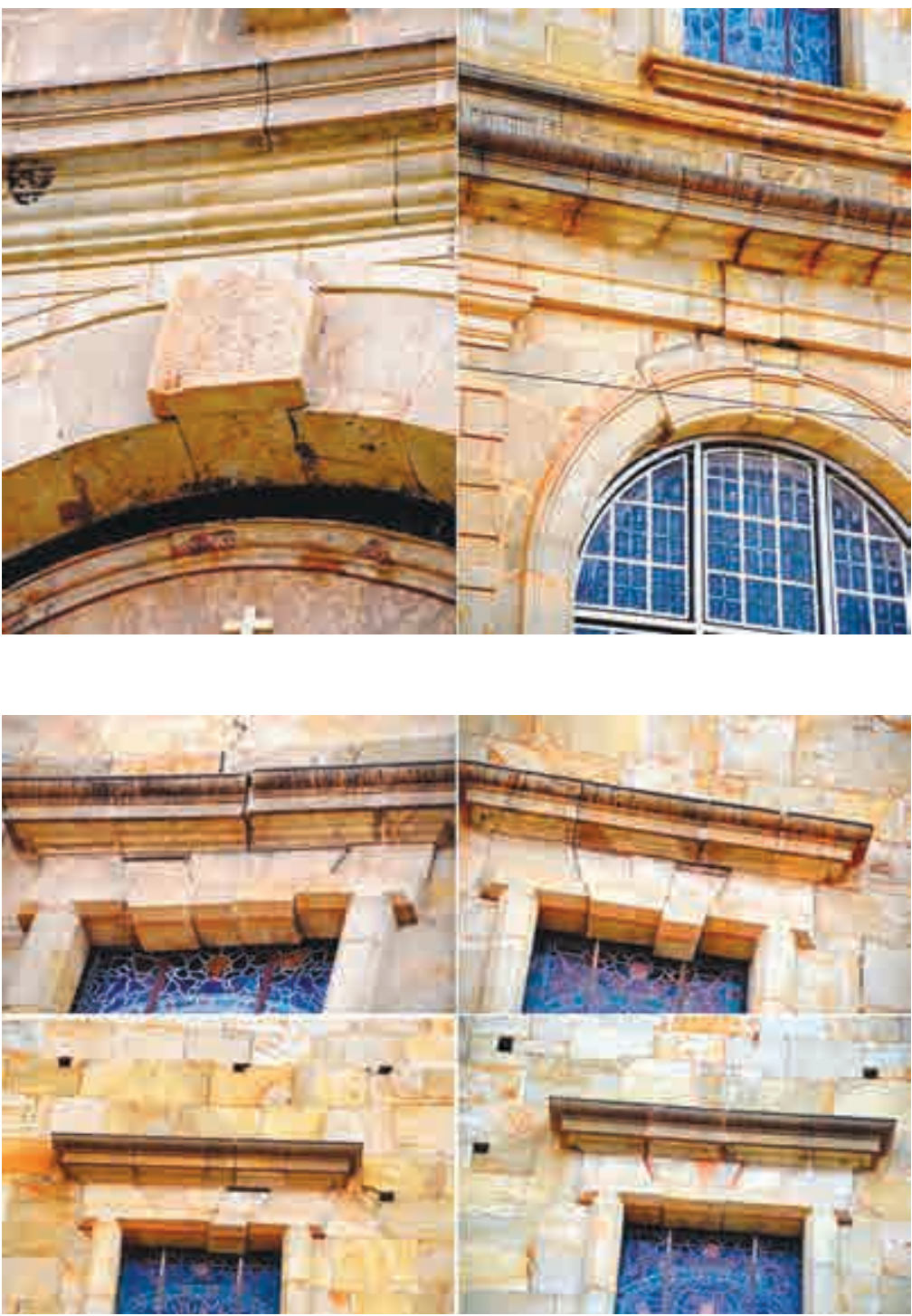

Nella sottostante figure 24, invece, si riporta lo stesso meccanismo di collasso ma per le architravi delle grandi finestrature della Basilica. Questi elementi strutturali, ancorché costruttivamente essere dellle piattabande, dal punto di vista strutturale si comportano, per la inclinazione delle sezioni tra concio e concio, come degli archi fortemente ribassati ed esercitano una azione fortemente spinfent sugli appoggi. In qualche misura questo meccanismo per cuneo è ancpra più pericoloso quando appaia nelle piattabande che non negli archi.
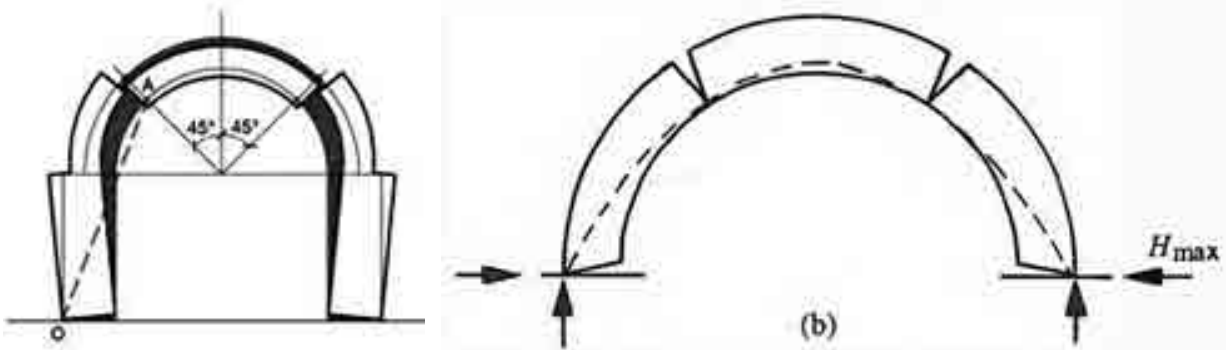
In letteratura tecnica si deve al fisico francese Coulomb, Charles Augustin de Coulomb ( I 736- I 806) a formalizzazione dei meccanismo di collasso degli archi e degli elementi costruttivi assimilabili ad essi . I meccanismi di collasso sono di due tipi: il meccanismo flessionale, che evolve l'arco da struttura tre volte iperstatica a monolabile e che è causato da eccessive azioni esterne di tipo statico, e quello per cuneo, caratterizzato dallo scivolamento verso il basso di parti dell'arco nella zona della chiave e dovuto a cedimenti degli appoggi e quindi ad azioni di tipo cinematico (Benvenuto, 198I), (Paradiso, Tempesta, Pugi, Galassi, 2000).

Per tutto quanto detto, la causa del quadro fessurativo, che in letteratura tecnica e definito di pre-crollo e quindi altamente pericoloso, è da ascriversioi a cedimenti fondazionali, come causa principale, ma certamente corroborata dalle continue azioni sismiche, da una probabile e storicizzata mancanza di attenzione alla manutenzione fisica dell'edificio monumentale, alle piogge, a una scarsa conoscenza del sottosuolo, alla totale mancanza di catene di ferro utili a contenere le spinte dei sistemi voltati e a fare opera di ammorsamento tra loro delle murature perimetrali. Nello schizzo finale della figure sottostante si evidenzia, molto schematicamente, la possibile situazione di non-ritorno.

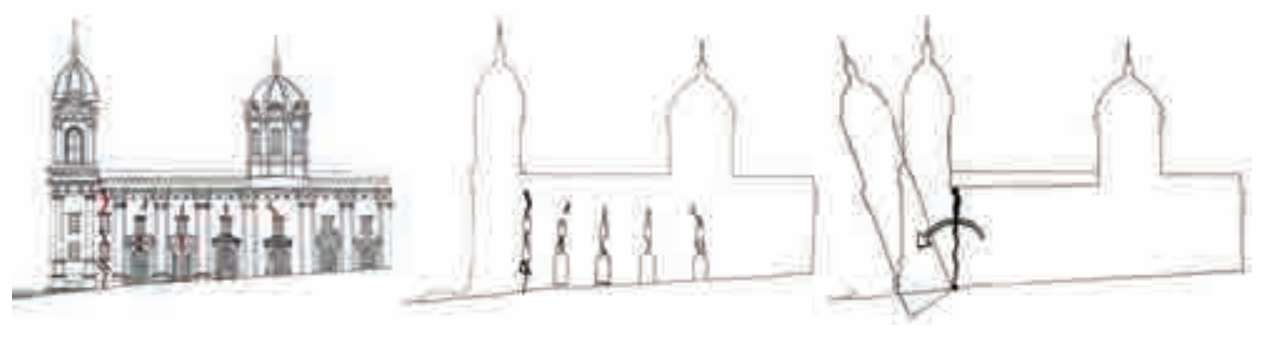

Tutto quello che si è fin qui descritto risale alla situazione del Monumento alla data del Marzo 2017. In quella occasione il Prof. Michele Paradiso, del Dipartimento di Architettura dell'Università degli Studi di Firenze-Italia, si trovava come visiting professor alla Facoltà di Architettura delle Universidad Santo Tomás de Bucaramanga e fu invitato dalla Comision de Monumentos del Departamento de Santander a fare un sopralluogo a Socorro perché le autorità locali erano preoccupate per l'aggravarsi del quadro fessurativo, noto già da parecchi anni e ulteriormente aggravatosi negli ultimi mesi del 2016 (Vanguardia Liberal, 2016), in conseguenza di recenti temblores e forti piogge. II sopralluogo, insieme ai Professori componenti la Linea de Patrimonio della UstaBuca, ebbe luogo domenica 19 marzo 2017. Alla fine del sopralluogo i componenti della missione esortarono vivamente la locale Protezione Civile a chiudere immediatamente al traffico pedonale e veicolare la Calle 14, per il pericolo che qualcuna delle grosse pietre degli archi e degli architravi che stavano sfilandosi dalla loro sede potessero cadere rovinando sulla strada sottostante. Non passarono 24 ore che la profezia si avverò: una grande pietra scivolata già verso il basso, del peso di $200 \mathrm{~kg}$, precipitò nella strada sottostante, per fortuna senza causare danni alle persone (Vanguardia Liberal, 20 I 7). La stampa locale dette molta eco alla cosa (Vanguardia Liberal, 2017) e fu immediatamente organizzata una riunione a Socorro tra i componenti del sopralluogo del 19 marzo, l'allora Secretario de Cultura del Departamento de Santander Arq. Oscar Villabona, e le autorità ecclesiastiche e laiche locali (Vanguardia Liberal, 2017). Ne nacque un ampio dibattito dal quale emerse che già nel 2013 la situazione era preoccupante tanto che fu dato mandato di redarre un progetto di messa in sicurezza del monumento, un progetto di consolidamento, che avrebbe avuto la rapida approvazione da parte del Ministerio de Cultura. Questo progetto, a firma dell'Ing. José Maria Navarro, era basato su una serie di indagini, peraltro pco approfondite in relazione alla caratterizzazione meccanica dei materiali in sito, risente in ogni suo passaggio del tipico enfoque hormigonista
Figure 26. Evoluzione del quadro fessurativo Fonte: Elaborazioni grafiche Olimpia Lotti e Antonio Farigu. 
di cultura ispanica-latinamericana, per il quale non si fa alcuna distinzione, nel valutare o progettare la sicurezza sismica di un edificio, tra una torre in cemento armato di 50 piani e una chiesina esile ma preziosa del secolo XVI. II progetto considerava sufficiente realizzare un reticolo alla quota subfondazionale per collegare le fondazioni storiche fra loro attraverso un sistema di travi in cemento armato. Ma fu scoprire in quella riunione che quel progetto del 2013 , alla data del marzo del 20 I7, non era stato ancora approvato, per ragioni intuibili ma non certe. In quella riunione però, da una parte fu preso l'impegno da parte del Ministerio de Cultura di aggiornare quell'antico progetto, alla luce del peggioramento della situazione, e di provvedere nel giro di un mese alla messa in sicurezza della Basilica con una serie di puntellamenti. Da parte della componente italiana fu suggerito di mettere in atto un sistema di monitoraggio attivo del quadro fessurativo. Nonostante gli accordi presi, le autorità ecclesiastiche locali pensarono bene di risolvere il problema della messa in sicurezza affidandosi a un artigiano locale il provvide a rimettere in situ il pietrone crollato, ammosandolo alle pietre adiacenti con delle barre di acciaio (Vanguardia Liberal, 2017). II che permise senz'altro che la Basilica non si chiudesse al culto per le imminenti celebrazioni della Settimana Santa, ma rendendo i tre pietroni, prima semplicemente legati da malta. un unico blocco, si alterò la morfologia degli elementi strutturali componenti il tessuto murario con la conseguenza che i risultati di un eventuale monitoraggio attivo del quadro fessurativo sarebbe risultato falsato.

\section{CONCLUSIONI}

Alla data della composizione di questo articolo, agosto 2018 , non risulta che sia stato fatto nulla di quanto emerso dalla riunione e dai relativi accordi del 25 marzo del 2017. Le ragioni, tristemente molto comuni e usuali, si possono leggere in (Vanguardia Liberal, 20 I7): controversie ideologiche e di opportunità del momento, mancanza di una strategia condivisa, insufficienze di preparazione tecnica. E non se ne capisce la ragione di questo gioco delle parti che porta all'immobilismo e certo non al bene del Monumento, in realtà qualcosa è stato fatto: le autorità eccelsiastiche locali, forti di quanto dichiarato alla stampa (l'edificio non è a rischio, le lesioni ci sono sempre state, eé probabile che crolli qualcosaltro prima della Basilica...) hanno provveduto, nello spirito di quanto dichiarato al momento della elevazione della Concattedrale a Basilica Minore, ad opere di imbellecimiento che dal punto di vista tecnico hanno significato, per l'ennesima volta, la semplice stuccatura delle lesioni, come se questo intervento, valido internazionalmente solo quando le lesioni sono ampie meno di un millimetro, potesse risolvere un problema strutturalmente tanto grave (Fig. 27). La stessa cosa è successa, nello stesso arco temprale (20I7-20I8) alla Chiesa di Barichara, che soffriva e soffre, simile a quella di Socorro per tipologia, posizione, materiali, etc... della stesso genere di problemi. E, prima ancora, alla Chiesa di Santa Lucia di Guane, dove nel 20I I esisteva un quadro fessurativo che aveva fatto completemante distaccare una Cappella laterale dal corpo di fabbrica centrale. Stesse cause (piogge e terremoti), stessa mancanza di una strategia di conservazione, stessa incomunicabilità tra le istituzioni preposte alla Salvaguardia dei Beni Culturali (Stato e Chiesa, Centri di Alta Formazione).

Non sono queste le indicazioni che danno le Carte Internazionali del Restauro di lcomosUnesco, peraltro accettate dalla Colombia col suo Comitato Nazionale Icomos-Colombia. Esiste in America Latina tutta una lentezza nell'acquisire la coscienza che la Conservazione del proprio Patrimonio Storico Costruito è una arma fondamentale per il dialogo tra Società Civile e Istituzioni. Quindi è per ridurre questa lacuna che bisogna lavorare, formando una coscienza, accompagnata da una preparazione culturale e tecnica con una visione internazionale del solving problem, a partire dai livelli educativi inferiori (Colegios...) fino alla formazione Universitaria, rinforzando o fondando nelle Scuole d'Architettura e non solo, uno specifico curriculum in Conservazione dei Beni Culturali Artistici ed Architettonici. L'Assemblea Generale Icomos di Firenze, del 2014, ha emanato una Dicharazione in cui si stabilisce che la Conservazione 


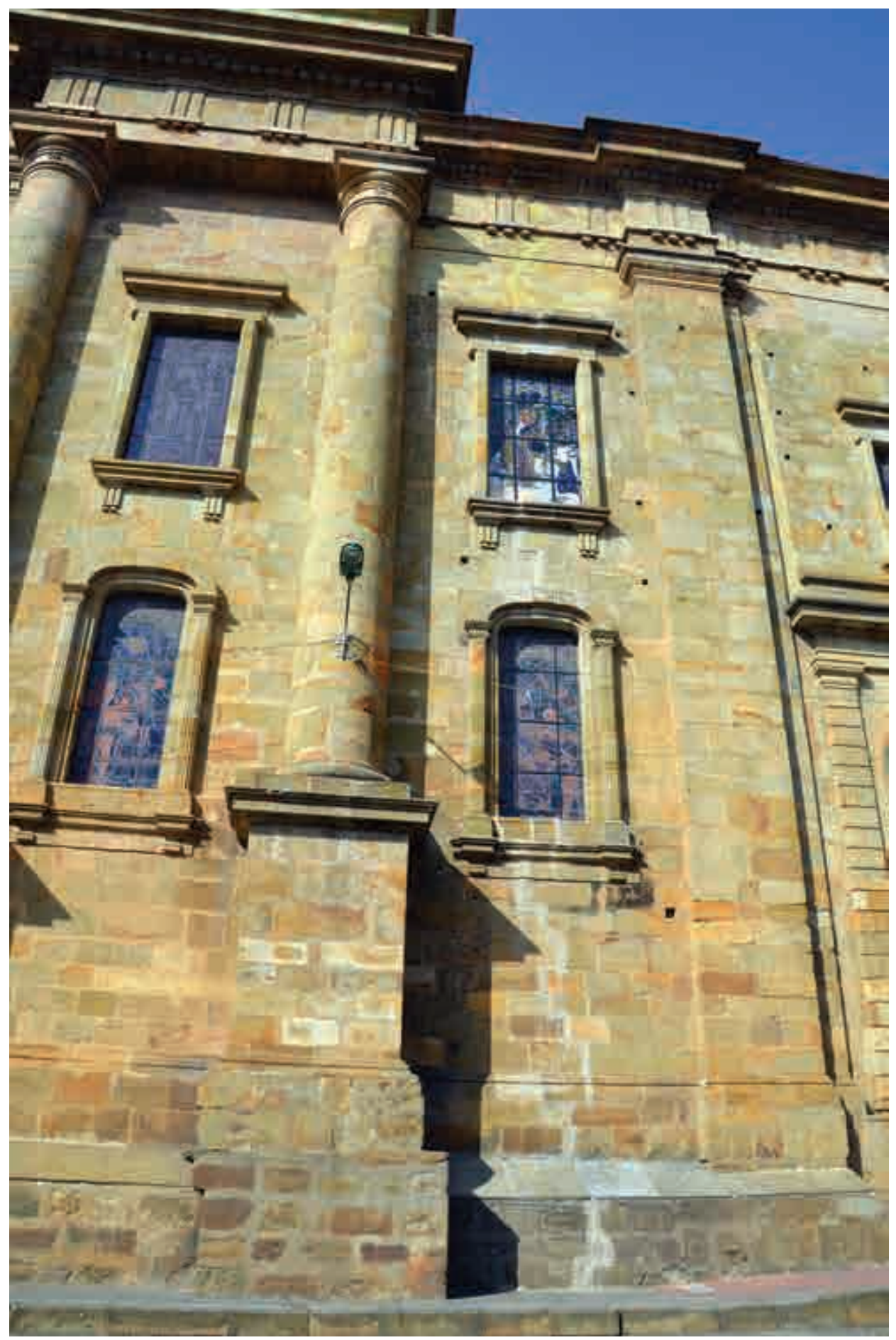

Figure 27. Stuccatura delle lesioni della facciato laterale della Basilica di Socorro Fonte: Michele Paradiso, 2018. 
e Salvagardia del Patrimonio Storico è un Valore Umano, quasi un diritto dell'uomo, mentre la recente Assemblea Generale di Nuova Delhi del 2018 ha avuto come titolo "Patrimonio e Democrazia". Proteggere la propria storia è un valore universale, al quale tutti abbiamo il dovere di concorrere, con uno sguardo non rivolto al tornaconto immediato, ma a una progetto di sviluppo che non dimentichi mai la storia. In questo, più o meno dappertutto nel mondo, la volontà politica, di qualsiasi segno o religione sia, è colpevolmente mancante.

Valga per tutti il monito di Victor Hugo;

\author{
Ci sono due cose in un edificio: il suo uso e la sua bellezza. \\ Il suo uso appartiene al proprietario, la sua bellezza al mondo intero. \\ Sicché permettere che l'edificio si distrugga va molto oltre il diritto del proprietario.
}

\title{
REFERENZE
}

Alcaldía de Socorro. (2018). Sitio web oficial. Recuperado de http://www.socorrosantander.gov.co/.

Arciniegas, G. (1992). Los Comuneros. Bogotá: Ed. ABC.

Benvenuto, E. (198I). La scienza delle costruzioni e il suo sviluppo storico. (Prima edizione). Roma: Edizioni di Storia e Letteratura.

Congreso de Colombia. Ley 53 de 192 7. Publicado en el Diario Oficial. Año. LXIII. N. 20644. 14 de noviembre de 1927, p. I. Recuperado de www.suin-juriscol.gov.co/clp/ contenidos.dll/Leyes/ $/ 60668 \mathrm{I}$ ? $\mathrm{n}=$ document-frame.htm $\$ \mathrm{f}=$ templates $\$ 3.0$

Mastrodicasa, S. (nona edizione, 20I2). Dissesti statici delle strutture edilizie. Milano: Ed. Hoepli.

Paradiso M., Tempesta G., Pugi, F., Galassi, S. (2000). Sistemi Voltati in muratura: teoria e applicazioni. Roma: Ed. DEl-Tipografia del Genio Civile.

Parra, A. G. (1955). Reseña histórica sobre la catedral de la ciudad del Socorro: apuntaciones tomadas de los archivos parroquiales. Socorro: Impr. Divino Niño.

Proyecto de Ley 267 de 20 I I Senado. Por la cual se declara bien de interés cultural de la Nación la Concatedral de Nuestra Señora del Socorro, ubicada en el municipio de Socorro, departamento de Santander y se dictan otras disposiciones. Recuperado de http://www. imprenta.gov.co/gacetap/gaceta.mostrar_documento?p_tipo $=05 \&$ __numero $=267 \&$ p_con$\mathrm{sec}=28992$

Vanguardia Liberal. (2016). Se desplazaron piedras de los arcos de la Basílica del Socorro. Recuperado de http://www.vanguardia.com/santander/comunera/379734-se-desplazaronpiedras-de-los-arcos-de-la-basilica-del-socorro.

Vanguardia Liberal. (2016). Departamento y municipio deberán apropiar recursos. Recuperado de http://www.vanguardia.com/santander/comunera/3802 I2-departamentoy-municipio-deberan-apropiar-recursos

Vanguardia Liberal. (2017). Intervendrán de "urgencia" la Basílica Menor del Socorro. Recuperado de http://www.vanguardia.com/santander/comunera/3900I6-intervendrande-urgencia-la-basilica-menor-del-socorro 
Vanguardia Liberal. (2017). Enormes piedras a punto de desprenderse de Basílica del Socorro. Recuperado de http://www.vanguardia.com/santander/comunera/392546enormes-piedras-a-punto-de-desprenderse-de-basilica-del-socorro

Vanguardia Liberal. (2017). Problemas estructurales en la Basílica Menor del Socorro. Recuperado de http://www.vanguardia.com/santander/comunera/392586-problemasestructurales-en-la-basilica-menor-del-socorro

Vanguardia Liberal. (2017). Declaran estado de alerta por fallas estructurales del templo. Recuperado de http://www.vanguardia.com/santander/comunera/392797-declaran-estadode-alerta-por-fallas-estructurales-del-templo

Vanguardia Liberal. (2017). Iniciaron intervención de la Basílica Menor. Recuperado de http://www.vanguardia.com/santander/comunera/392693-iniciaron-intervencion-dela-basilica-menor

Vanguardia Liberal. (2017). Iglesia asegura que no tiene \$7 mil millones para arreglar Basílica del Socorro. Recuperado de http://www.vanguardia.com/santander/comunera/394537iglesia-asegura-que-no-tiene-7-mil-millones-para-arreglar-basilica-del-soc 